\section{Stellar photospheres}

The Observation and Analysis of Stellar Photospheres. By David F. Gray. Pp. $x v+471$. (Wiley-Interscience: New York and London, January 1976.) $\$ 27.70 ; £ 14$.

THE study of stellar atmospheres (or even of just their photospheres, which are the layers emitting the bulk of the radiation) has become too vast a subject to be comprehensively covered in depth in a single book, and so it is natural (and indeed desirable) to find authors concentrating on their own particular fields of research interest while summarising other aspects more briefly. The distinctive and very valuable feature of the present book is that it provides a detailed elementary introduction to the experimental measurement and analysis of stellar spectral characteristics using predominantly Fourier techniques-a subject in which the author has himself done some very useful work and which is coming to be an essential tool in all spectral analysis with the advent of high speed multichannel detectors and digital microdensitometers.

In other respects, the book is a clear, well written and exceptionally well illustrated introduction from the practical point of view with enough of the theory of stellar atmospheres given (accompanied by many numerical formulae and tables) to show the application to the determination of effective temperature, pressure, gravity, radius, chemical composition and velocity fields. Difficult theoretical issues, like the intricacies of iteration to flux constancy and the effect of departures from local thermodynamic equilibrium, are mentioned and summarised as accurately as is possible within a few words supplemented by references, which is quite suitable for a text of finite size. It is always a weakness in this kind of treatment, however, that the reader does not quite get a physical feeling for the subject.

The presence of a few minor errors in places (for example, in the treatment of inward-directed radiation in the theory of radiative transfer) does not help and on these matters the reader will do well to consult both the old classical texts of Woolley and Stibbs, Unsöld and Aller and the modern one by Mihalas. There is, however, a very fine discussion (not otherwise available in textbooks or perhaps anywhere else) of the use of line profiles to sort out rotation and different sorts of 'turbulence'-a field in which the author's Fourier methods offer an enticing vista of new research.

In the spirit of the generally practical approach of this book, there are extensive sections on spectrometers and detectors. These are generally very good, although it is a matter for some regret that the roles of grating blaze angle and camera speed in the efficiency of spectrographs have not been explicitly brought out. The treatment of instrumental profile and scattered light corrections is excellent.

Particularly in its treatment of the practical aspects of stellar spectrum analysis, this is a valuable book that is strongly recommended for all astronomical libraries and could be a worthwhile investment for research workers and graduate students; the price is not too unreasonable in present-day conditions.

Bernard Pagel

Bernard Pagel is Deputy Chief Scientific Officer at the Royal Greenwich Observatory and Visiting Professor at the Astronomy Centre, University of Sussex, UK.

\section{Membrane research}

Mammalian Cell Membranes. Vol. 1 : General Concepts. Edited by G. A. Jamieson and D. N. Robinson. Pp. 276. (Butterworth : London and Boston, Massachusetts, 1976.) $£ 18$.

IT has been said that a week is a long time in politics and things are becoming almost as fluid in membrane research. Since 1973, for instance, when much of the material in this book was prepared, the complete sequence of a transmembrane protein has been established by Marchesi and his colleagues. The molecular basis of ion conductance across membranes has become clearer at least conceptually in artist's impressions if not in hard fact, and the proteins involved are being characterised. Similarly the importance of microfilaments and microtubules in membrane function in non-muscle cells has been realised.

Some of the treatment understandably has a slightly old-fashioned look. For example, in Rose's short chapter on the anatomy of the mammalian ccll discussion of the various forms of junctional complexes in cells must be inadequate in view of the considerable advances made on their structure and function. Two other examples concern surface labelling procedures particularly with lactoperoxidase-catalysed iodination and SDS-polyacrylamide gel clectrophoresis. The description is very scanty considering the recent extensive use of these techniques.

The book is the first in a five-volume series, and according to the Editors is an introduction to "the most essential aspects of physical and chemical studies which have contributed to present knowledge of membrane structure." This volume, however, is sharply divided on the basis of these criteria. On the one hand there are clear and straightforward descriptions, respectively, of the cultivation of cells by Robinson and a brief account by Glick of selected methods of plasma membrane preparation largely from cultured cells. Physical methods are covered by Levine and Chapman, and enzyme markers are summarised in a useful chapter by Hinton and Reid. The remainder of the book consists of four short speculative chapters on specialised, even esoteric, topics. Gingell argues for the electrostatic control of diffusion of proteins in membranes. The basic point is that proteins carry high negative charges (mainly due to sialic acid groups) that must be overcome by ligand binding before condensation can occur. Condensation of transmembrane proteins, equated by Gingell with intramembranous particles, is considered to be obligatory for formation of ion channels through the membrane. Well, maybe; but hardly the basic facts required for an introduction to membrane science. One difficulty too is the as yet inconclusive evidence, except in erythrocytes, that intramembranous particles can be clustered by ligand interaction with surface membrane proteins.

The infant science of the mechanical properties of cellular membranes is interestingly reviewed by Canham, and Lenard and Landsberger discuss some models of membrane structure. The biogenesis of mammalian membranes is summarised by Malhotra. I enjoyed reading these four chapters and, for my money, they recommend the book for this year's bookshelf.

On the whole each chapter is well written and free of serious factual or typographical errors. The book should be useful to research workers and to final year undergraduates. The person who discovers on p32 that trypsin "catalyses the hydrolysis of peptide bonds between the carboxy group of arginine or lysine and the amino group of an adjacent amino acid" is likely to have difficulty in following many of the complex arguments that follow elsewhere in the book. The editors are to be congratulated on setting a good standard for the first volume and I look forward to the arrival of the remaining volumes. $\quad$ R. Colin Hughes

R. Colin Hughes is a member of the scientific staff of the Medical Research Council at the National Institute for Medical Research, Mill Hill, London, UK. 